\title{
ÜBER DIE ASYMPTOTISCHE DARSTELLUNG DER INTEGRALE
}

\section{LINEARER DIFFERENTIALGLEICHUNGEN}

voN

\section{J. HORN}

in CLAUSTHAL.

Im ersten Theil meiner Arbeit Über das Verhalten der Integrale von Differentialgleichungen bei der Annäherung der Veründerlichen an eine Unbestimmtheitsstelle ${ }^{1}$ habe ich das Verhalten der Integrale einer Riccati'schen Differentialgleichung und einer linearen Differentialgleichung zweiter Ordnung für den Fall untersucht, dass die unabhängige Veränderliche auf einem bestimmten Wege nach einer Unbestimmtheitsstelle geht. Für die lineare Differentialgleichung zweiter Ordnung, ${ }^{2}$ deren Coefficienten in der Umgebung der Unbestimmtheitsstelle den Charakter rationaler Functionen haben, habe ich die Sätze des Herrn PorNcalé ${ }^{3}$ über die asymptotische Darstellung der Integrale linearer Differentialgleichungen mit rationalen Coefficienten durch die Thoméschen Normalreihen ohne Benutzung der Laplace'schen Transformirten bewiesen und vervollständigt, indem ich an die Untersuchung des Grenzwerthes der logarithmischen Ableitung am Anfang der Abhandlung des Herrn PoIscaké im American Journal anknüpfte. Damals kam es mir vorzugsweise darauf an, Methoden zu gewinnen, welche sich, wie der zweite und dritte Theil ${ }^{4}$ der angeführten

1 Crelles Journal Bd. I 18.

2 Vgl. auch KNeser, Untersuchung und asymptotische Darstellung der Integrale gewisser Differentialgleichungen bei grossen reellen Werthen des Arguments (Crelles Journ. Bd. I 16 u. 1 1 7).

3 American Journ. Bd. 7, Acta math. Bd. 8.

Crelles Joura. Bd. I I9. Vgl. die Arbeiten von Herro Bendixson. 
Arbeit zeigen, auf nicht lineare Differentialgleichungen übertragen lassen. Indem ich jetzt diese Rücksicht bei Seite lasse, beweise ich für eine lineare Differentialgleichung $n^{\text {ter }}$ Ordnung, deren Coefficienten in der Umgebung der Unbestimmtsheitsstelle $x=\infty$ den Charakter rationaler Functionen haben, ${ }^{1}$ die asymptotische Darstellung der Integrale durch die der Differentialgleichung formell genügenden divergenten Reihen, wobei ich wie in der oben erwähnten Arbeit vorläufig noch einzelne nach der Stelle $x=\infty$ führende Wege ausschliesse und die Wurzeln der charakteristischen Gleichung als verschieden voraussetze. Auch jetzt mache ich von der Laplace'schen Transformirten keinen Gebrauch, sondern nur von Poincaré's Untersuchung des Grenzwerthes der logarithmischen Ableitung und von der bekannten Erniedrigung der Ordnung einer linearen Differentialgleichung unter Benutzung eines particulären Integrals.

\section{$\S \mathbf{I}$.}

Wir schicken einige Hilfsuntersuchungen voraus. ${ }^{2}$

In dem Differentialgleichungssystem

$$
x^{-k} \frac{d w_{\lambda}}{d x}=\alpha_{\lambda} w_{\lambda}+\sum_{\mu} Q_{\lambda \mu} w_{\mu} \quad(\lambda, \mu=1, \ldots, m)
$$

sei $k$ eine ganze positive Zahl (einschl. o), die $Q_{\lambda \mu}$ Functionen von $x$ mit der Eigenschaft

$$
\lim Q_{\lambda \mu}=\mathrm{o}^{3}
$$

und $\alpha_{1}, \ldots, \alpha_{m}$ constante Grössen, deren reelle Theile eine absteigende Reihe bilden und sämmtlich verschieden sein sollen. Wir lassen $x$ als

${ }^{1}$ Die Untersuchungen des Herrn Poincaré, welche sich auf die Laplace sche Trans. formation gründen, setzen die Coefficienten als rational in der ganzen Ebene voraus, während hier nur das Verhalten in der Uagebung der Unbestimmtheitsstelle in Frage kommt.

2 Vgl. Poncaré, Am. Journ. Bd. 7.

${ }^{3}$ Unter $\lim f(x)$ wird stets der Grenzwerth verstanden, welchem die Function $f(x)$ zustrebt, wenn $x$ als reelle positive Grösse ins Unendliche geht. 
Über die asymptotische Darstellung der Integrale linearer Differentialgleichungen. 291 reelle positive Grösse ins Unendliche gehen und untersuchen das Verhalten der Quotienten

$$
\frac{w_{\lambda}}{w_{1}} \cdot \quad(\lambda=2, \ldots, m)
$$

Es ist

$$
x^{-k} \frac{d \log \frac{w_{\lambda}}{w_{1}}}{d x}=\alpha_{\lambda}-\alpha_{1}+Q_{\lambda \lambda}-Q_{11}+\sum_{\mu \neq \lambda} Q_{\lambda \mu} \frac{w_{\mu}}{w_{\lambda}}-\sum_{\mu>1} Q_{1 \mu} \frac{w_{\mu}}{w_{1}} \quad(\lambda=2, \ldots, m)
$$

und

$$
R\left(\alpha_{\lambda}-\alpha_{1}\right)<0 .^{1}
$$

Wir haben

$$
\left|Q_{\lambda, \mu}\right|<o
$$

wo $\delta$ eine für $x=\infty$ verschwindende positive Grösse ist. Wenn man

$$
2 \delta\left(\mathrm{I}+\frac{m-1}{\varepsilon}\right)=R\left(\alpha_{1}-\alpha_{2}\right)-g
$$

setzt, wo $g$ eine kleine positive Grösse darstellt, so ist $\varepsilon$ für grosse $x$ positiv und

$$
\lim \varepsilon=\text { o. }
$$

Wenn für einen gewissen Werth von $x$

$$
\begin{array}{ll}
\left|\frac{w_{\mu}}{w_{\lambda}}\right|<\frac{I}{\varepsilon} & (\mu=1, \ldots, \lambda-1, \lambda+1, \ldots, m) \\
\left|\frac{w_{\mu}}{w_{1}}\right|<\frac{I}{\varepsilon} & (\mu=2, \ldots, m)
\end{array}
$$

ist, so ist der reelle Theil von

kleiner als

$$
x^{-k} \frac{d \log \frac{w_{\lambda}}{w_{1}}}{d x}
$$

$$
R\left(a_{2}-a_{1}\right)+2 \delta+2(m-\mathrm{I}) \dot{\partial} \cdot \frac{\mathrm{I}}{\varepsilon}
$$

also

$$
x^{-k} \frac{d \log \left|\frac{w_{\lambda}}{w_{1}}\right|}{d x}<-g .
$$

${ }^{1} R(a)$ ist der reelle Theil von $a$. 
Wir verstehen unter $\boldsymbol{M}$ den jeweilig grössten der Quotienten

$$
\left|\frac{w^{i}}{w_{1}}\right|
$$

und zeigen, dass, wenn

$$
\varepsilon<M<\frac{1}{\varepsilon}
$$

ist,

$$
x^{-k} \frac{d \log M}{d x}<-g
$$

sein muss. Es sei

$$
M=\left|\begin{array}{l}
w_{i} \\
w_{1}
\end{array}\right| \geq\left|\begin{array}{l}
w_{\lambda} \\
w_{1}
\end{array}\right| \geq \ldots
$$

Wegen

$$
M<\frac{\text { I }}{\varepsilon}
$$

ist

$$
\left|\frac{w_{1}}{w_{1}}\right|<\frac{\mathrm{I}}{\varepsilon}
$$

ferner ist

$$
\left|\frac{w_{1}}{w_{\lambda^{\prime}}}\right|<\frac{\mathbf{I}}{\varepsilon}
$$

wegen

$$
M=\left|\frac{w_{\lambda}}{w_{1}}\right|>\varepsilon
$$

und

$$
\left|\frac{w_{\mu}}{w_{\lambda^{\prime}}}\right|<\mathrm{I}<\frac{\mathrm{I}}{\varepsilon}
$$

wegen

$$
\left|w_{\lambda^{\prime}}\right| \geqq\left|w_{\lambda^{\prime \prime}}\right| \geqq \ldots ;
$$

es ist also

$$
\left|\frac{w_{\mu}}{w_{\lambda^{\prime}}}\right|<\frac{\mathbf{I}}{\varepsilon} .
$$


Über die asymptotische Darstellung der Integrale linearer Differentialgleichungen. 293

Unter diesen Voraussetzungen ist aber

$$
x^{-k}-\frac{d \log \left|\frac{w_{\lambda}}{w_{1}}\right|}{d x}=x^{-k} \frac{d \log M}{d x}<-g .
$$

Wenn für einen gewissen Werth von $x M$ zwischen $\varepsilon$ und $\frac{\mathrm{I}}{\varepsilon}$ liegt und bei wachsendem $x$ grösser als $\varepsilon$ bleibt, so ist hiernach

$$
\lim M=0
$$

das gleiche ist der Fall, wenn für beliebig grosse Werthe von $x \quad M \leq \varepsilon$ ist. Mit anderen Worten, wenn für einen grossen Werth von $x$

$$
\left|\frac{w_{\lambda}}{w_{1}}\right|<\frac{\mathrm{I}}{\varepsilon} \quad(\lambda=2, \ldots, m)
$$

ist, so ist

$$
\lim \frac{w_{\lambda}}{w_{1}}=0
$$$$
(\lambda=2, \ldots, m)
$$

Für das Folgende genügt es, zu wissen, dass überhaupt ein Integralsystem $w_{1}, \ldots, w_{m}$ des Differentialgleichungssystems (A) vorhanden ist, welches die Eigenschaft besitzt, dass sich sämmtliche Quotienten

$$
\frac{w_{2}}{w_{1}}, \ldots, \frac{w_{m}}{w_{1}}
$$

der Grenze Null nähern, wenn $x$ als reelle positive Grösse ins Unendliche geht.

Die Functionen

$$
z_{\lambda}=\frac{w_{\lambda}}{w_{1}}
$$

$(\lambda=2, \ldots, m)$

genügen den Differentialgleichungen

$$
x^{-k} \frac{d z_{\lambda}}{d x}+z_{\lambda} \sum_{\mu} R_{1 \mu} z_{\mu k}=\beta_{\lambda} z_{\lambda}+\sum_{\mu} R_{\lambda \mu} z_{\mu}+R_{\lambda_{1}}, \quad(\lambda, \mu=2, \ldots, m)
$$


wobei gesetzt ist:

$$
\begin{aligned}
& \beta_{\lambda}=\alpha_{\lambda}-\alpha_{1}, \\
R_{1 \mu}= & Q_{1 \mu}, \quad R_{\lambda 1}=Q_{\lambda 1}, \\
R_{\lambda \mu}= & Q_{\lambda \mu}, \quad(\lambda \neq \mu) \\
R_{\lambda \lambda}= & Q_{\lambda \lambda}-Q_{11} .
\end{aligned}
$$

Ein System von der Form (B) besitzt ein Integralsystem $z_{2}, \ldots, z_{m}$ mit der Eigenschaft

$$
\lim z_{\lambda}=\mathrm{O}, \quad(\lambda=2, \ldots, m)
$$

wenn

$$
R\left(\beta_{2}\right)<\mathrm{o}, \quad(\lambda=2, \ldots, m)
$$

$\lim R_{1 \mu}=$ o, $\quad \lim R_{\lambda 1}=$ o, $\quad \lim R_{\lambda, \mu}=\mathrm{o}$

ist. Denn ein solches System (B) kann aus einem System von der Form (A) hergeleitet werden.

Wir nehmen jetzt an, die Functionen $R_{\lambda \mu}(\lambda, \mu=\mathrm{I}, \ldots, m)$ seien von der Form

$$
\begin{gathered}
R_{\lambda \mu}=\frac{b_{\lambda \mu \mu}^{(1)}}{x}+\ldots+\frac{b_{\lambda \mu}^{(n)}}{x^{n}}+\frac{\beta_{\lambda \mu}^{(n)}}{x^{n}} \\
\lim \beta_{\lambda \mu}^{(n)}=\mathrm{o}
\end{gathered}
$$

wo $n$ eine feste ganze positive Zahl darstellt. Erzetzt man jede Function $\beta_{\lambda_{\mu k}}^{(n)}$ durch Null, so wird das System (B) durch ein System von Potenzreihen

$$
z_{\lambda}=\frac{c_{\lambda 1}}{x}+\frac{c_{\lambda 2}}{x^{2}}+\ldots
$$

$(\lambda=2, \ldots, m)$

formell befriedigt. Wir zeigen, dass das System (B) ein Integralsystem von der Form

$$
\begin{gathered}
z_{\lambda}=\frac{c_{\lambda 1}}{x}+\ldots+\frac{c_{\lambda n}}{x^{n}}+\frac{\zeta_{\lambda n}}{x^{n}}=z_{\lambda n}+\frac{\zeta_{\lambda n}}{x^{n}} \\
\lim \zeta_{\lambda n}=0
\end{gathered}
$$

besitzt. Die Functionen

$$
\zeta_{\lambda}=\zeta_{\lambda n}
$$


Über die asymptotische Darstellung der Integrale linearer Differentialgleichungen. 295 genügen nämlich den Differentialgleichungen

$$
x^{-\lambda} \frac{d \zeta_{\lambda}}{d x}+\zeta_{\lambda} \sum_{\mu} S_{\lambda \mu} \zeta_{\mu}=\beta_{\lambda} \zeta_{\lambda}+\sum_{\mu} S_{\lambda, \mu} \zeta_{\mu}+S_{\lambda 1}, \quad(\lambda, \mu=2, \ldots, m)
$$

wenn man setzt:

$$
\begin{aligned}
& S_{\mathrm{i} \mu}=\frac{R_{1 \mu}}{x^{n}}, \\
& S_{\lambda \mu}=R_{\lambda \mu}-R_{1 \mu} z_{\lambda \mu} \quad(\lambda \pm \mu), \\
& S_{\lambda \lambda}=R_{\lambda \lambda}-R_{1 \lambda} z_{\lambda n}-\left(R_{12} z_{2 n}+\ldots+R_{1 m} z_{m n}\right)+\frac{n}{x^{k+1}}, \\
& S_{\lambda 1}=\varphi_{\lambda 1},
\end{aligned}
$$

wo $\varphi_{\lambda 1}$ eine Function von $x$ mit

$$
\lim \varphi_{\lambda x}=0
$$

ist. Ein System von dieser Form besitzt aber, wie vorhin gezeigt wurde, ein Integralsystem $\zeta_{2}, \ldots, \zeta_{m}$ mit der Eigenschaft

$$
\lim \zeta_{\lambda}=0
$$$$
(\lambda=2, \ldots, n)
$$

$$
\S 2 .
$$

Den eigentlichen Gegenstand unserer Untersuchung bildet die Differentialgleichung

$$
\frac{d^{m} y}{d x^{m}}+x^{k} P_{1} \frac{d^{m-1} y}{d x^{m-1}}+x^{2 k} P_{2} \frac{d^{m-2} y}{d x^{m-2}}+\ldots+x^{(m-1) k} P_{m-1} \frac{d y}{d x}+x^{m k} P_{m} y=0
$$

Die Coefficienten

$$
P_{\lambda}=a_{\lambda}+\frac{a_{\lambda 1}}{x}+\frac{a_{\lambda 2}}{x^{3}}+\ldots
$$

sind entweder Potenzreihen von $\frac{1}{x}$, welche in der Umgebung von $x=\infty$ convergent sind, oder Functionen, welche durch divergente Potenzreihen von $\frac{I}{x}$ asymptotisch dargestellt werden, wenn $x$ als reelle positive Grösse 
ins Unendliche geht; in beiden Fällen ist, welche ganze positive Zahl auch für $n$ gesetzt werden möge,

$$
\begin{gathered}
P_{\lambda}=a_{\lambda}+\frac{a_{\lambda 1}}{x}+\ldots+\frac{a_{\lambda n}}{x^{n}}+\frac{\bar{\omega}_{\lambda n}}{x^{n}} \\
\lim \bar{\omega}_{\lambda n}=0 .
\end{gathered}
$$

Die charakteristische Gleichung von (C)

$$
\alpha^{m}+a_{\jmath} \alpha^{m-1}+\ldots+a_{m-1} \alpha+a_{m}=0
$$

habe $n$ verschiedene Wurzeln

$$
\alpha_{1}, \alpha_{2}, \ldots, \alpha_{m}
$$

deren reelle Theile verschieden und absteigend geordnet seien.

Setzt man ${ }^{1}$

$$
\begin{aligned}
y & =w_{1}+\ldots+w_{m} \\
x^{-\nu k} \frac{d^{\nu} y}{d x^{\nu}} & =\alpha_{1}^{\nu} w_{1}+\ldots+\alpha_{m}^{\nu} w_{m}, \quad \quad(\nu=1, \ldots, m-1)
\end{aligned}
$$

co genügen die Functionen $w_{1}, \ldots, w_{m}$ einem Differentialgleichungssystem von der Form (A), woraus durch die Substitution

$$
z_{\lambda}=\frac{w_{\lambda}}{w_{1}} \quad(\lambda=2, \ldots, m)
$$

ein System von der Form (B) hervorgeht. Es ist

$$
R\left(\beta_{\lambda}\right)=R\left(\alpha_{\lambda}-a_{1}\right)<0 \quad(\lambda=2, \ldots, m)
$$

und entweder

$$
R_{\lambda \mu}=\frac{b_{\lambda \mu}^{(1)}}{x}+\frac{b_{\lambda \mu}^{(2)}}{x^{2}}+\ldots \quad(\lambda, \mu=1, \ldots, m)
$$

eine convergente Potenzreihe oder

$$
\begin{gathered}
R_{\lambda \mu}=\frac{b_{\lambda \mu}^{(1)}}{x}+\ldots+\frac{b_{\lambda \mu}^{(n)}}{x^{n}}+\frac{\beta_{\lambda \mu}^{(n)}}{x^{n}} \\
\lim \beta_{\lambda \mu}^{(n)}=0
\end{gathered}
$$

${ }^{1}$ Vgl. Poincare, Am. Journ. Bd. 7 . 
Üler die asymptotische Darstellung der Integrale linearer Differentialgleichungen. 297 fïr jedes $n$. Setyt man für jede Function $R_{n^{\prime \prime}}$ die convergente oder asymptotische unendliche Reihe, so wird das System (B) durch das Reihensystem

$$
z_{\lambda}=\frac{c_{\lambda 1}}{x}+\frac{c_{\lambda 2}}{x^{2}}+\ldots \quad(\lambda \approx 2, \ldots, m)
$$

formell befriedigt. Nimmt man jetzt für $n$ eine feste Kahl, so besitzt (B), wie in $\$$ I gezeigt wurde, eine Liösung

$$
\begin{gathered}
z_{\lambda}=\frac{c_{\lambda 1}}{x}+\ldots+\frac{c_{\lambda n}}{x^{n}}+{ }_{x^{n}}^{\zeta_{n n}}, \\
\lim \zeta_{\lambda, n}=0 .
\end{gathered}
$$

Ob diese lüsung auch für jede Zahl $n^{\prime}>n$ auf die Form

$$
z_{\lambda}==\frac{c_{\lambda 1}}{x}+\ldots+\frac{c_{\lambda n^{i}}}{x^{n^{\prime}}}+\frac{\dot{s i n}^{\prime}}{x^{n^{\prime}}}, \quad \lim \zeta_{i, n^{\prime}}=0
$$

gebracht werden kann, d. h. ob die Function $z_{\text {. }}$ durch die Reihe

$$
\frac{c_{\lambda 1}}{x}+\frac{c_{\lambda 2}}{x^{2}}+\ldots
$$

asymptotisch dargestellt wirl, bleibt noch dahin gestellt.

Aus den formalen Reihenentwicklungen

$$
z_{2}=\frac{c_{\lambda 1}}{x}+\frac{r_{\lambda 2}}{x^{2}}+\ldots
$$

ergibt sich der Gleichung

$$
\begin{aligned}
x^{-k} \frac{d \log !}{d x} & =\frac{\alpha_{1} w_{1}+\ldots+\alpha_{m} w_{m}}{w_{1}+\ldots+w_{m}} \\
& =\frac{\alpha_{1}+\alpha_{2} z_{2}+\ldots+\alpha_{m} z_{m}}{\mathrm{I}+z_{2}+\ldots+z_{m}}
\end{aligned}
$$

zufolge die formale Entwickelung

$$
x^{-k} \frac{d \log y}{d x}=a_{1}+\frac{\alpha_{11}}{x^{2}}+\frac{\alpha_{12}}{x^{2}}+\frac{\alpha_{13}}{x^{3}}+\ldots
$$

oder, wenn man

$$
\begin{gathered}
\alpha_{1, k+1}=\rho_{1}, \\
e^{-\frac{\alpha_{1, k+2}-\frac{a_{1, k+3}}{x}-\ldots}{2 x^{2}}-\cdots}=C_{1}+\frac{C_{11}}{x}+\frac{C_{13}}{x^{2}}+\ldots
\end{gathered}
$$

Acta mathematica. 24. Imprimé le 5 octobre 1900. 
setzt, die der Differentialgleichung (C) formal genügende Normalreihe

$$
y=S_{1}=e^{\frac{a_{1} x^{k+1}}{k+1}+\frac{a_{11} x^{k}}{k}+\ldots+a_{1 k} x} x^{\rho_{1}}\left(C_{1}+\frac{C_{12}}{x}+\frac{C_{12}}{x^{2}}+\ldots\right)
$$

Aus der Gleichung

folgt

$$
\begin{gathered}
z_{\lambda}==\frac{c_{\lambda I}}{x}+\ldots+\frac{c_{\lambda, n+k+1}}{x^{n+k+1}}+\frac{\zeta_{\lambda, n+k+1}}{x^{n+k+1}}, 1 \\
\lim \zeta_{\lambda, n+k+1}=0
\end{gathered}
$$

$$
\begin{gathered}
x^{-k} \frac{d \log y_{1}}{d x}=\frac{\alpha_{1}+\alpha_{2} z_{2}+\ldots+\alpha_{m} z_{m}}{1+z_{2}+\ldots+z_{m}}=\alpha_{1}+\frac{\alpha_{11}}{x}+\ldots+\frac{\alpha_{1, n+k+1}}{x^{n+k+1}}+\frac{\eta_{1 n}}{x^{n+k+1}}, \\
\lim \eta_{1 n}=0
\end{gathered}
$$

oder

$$
\begin{gathered}
y_{1}=e^{\frac{a_{1} x^{k+1}}{k+1}+\frac{a_{11} x^{k}}{k}+\ldots+\alpha_{1 n} x} x^{\rho_{1}}\left(C_{1}+\frac{C_{11}}{x}+\ldots+\frac{C_{1 n}}{x^{n}}+\frac{\gamma_{1 n}}{x^{n}}\right) \\
\lim \gamma_{1 n}=0 .
\end{gathered}
$$

Da $n$ eine zwar von vornherein willkürlich gewählte, aber dann festgehaltene Zahl ist, so ist noch nicht bewiesen, dass die Function $y_{1}$ durch die Reihe $S_{1}$ asymptotisch dargestellt wird.

Aus der Gleichung

$$
x^{-\nu k} \frac{\frac{d^{\nu} y_{1}}{d x^{\nu}}}{y_{1}}=\frac{\alpha_{1}^{\nu}+u_{2}^{\nu} z_{2}+\ldots+\alpha_{m}^{\nu} z_{m}}{\mathrm{I}+z_{2}+\ldots+z_{m}}
$$

folgen noch Gleichungen von der Form

$$
\begin{gathered}
x^{-\nu k} \frac{\frac{d^{\nu} y_{1}}{d x^{\nu}}}{y_{1}}=\alpha_{1}^{\nu}+\frac{\alpha_{11}^{(\nu)}}{x}+\ldots+\frac{\alpha_{1, n+k+1}^{(\nu)}}{x^{n+k+1}}+\frac{\eta_{n n}^{(\nu)}}{x^{n+k+1}}, \\
\lim \eta_{1 n}^{(\nu)}=0
\end{gathered}
$$

1 Unter $z_{2}, \ldots, z_{m}$ wird das oben eingefuhrte Integralsystem von (B) verstanden; es ist nur dic Bezeichnung insofern geändert, als die beliebige, aber feste Zahl $n$ jetzt wit $n+k+$ I bezeichnet ist. 
Über die asymptotische Darstellung der Integrale linearer Differentialgleichungen. 299 und insbesondere

$$
\lim x^{-\nu k} \frac{\frac{d^{\nu} y_{1}}{d x^{\nu}}}{y_{1}}=\alpha_{1}^{\nu} . \quad(\nu=1, \ldots, m-1)
$$

\section{$\S 3$.}

Die am Anfang von $\$ 2$ beschriebene Differentialgleichung (C) wird formell befriedigt durch $m$ Normalreihen

$$
S_{\lambda}=e^{\frac{a_{\lambda} x^{k+1}}{k+1}+\frac{a_{\lambda} x^{k}}{k}+\ldots+\alpha_{\lambda . k} x} x^{\rho \lambda}\left(C_{\lambda}+\frac{C_{\lambda \cdot}}{x}+\frac{C_{\lambda .2}}{x^{2}}+\ldots\right) . \quad(\lambda=1, \ldots, m)
$$

Wir bewiesen den folgenden Satz: ${ }^{1}$

Die Differentialgleichung (C) besitzt ein Fundamentalsystem

$$
y_{1}, \ldots, y_{m}
$$

von der Eigenschaft, dass die Function $y_{\lambda}$ durch die Normalreihe $S_{\lambda}$ asymptotisch dargestellt wird, wenn $x$ als reelle positive Grösse ins Unendliche geht; d. h. es ist, wenn $n$ irgend eine ganze positive Zahl ist,

$$
\begin{aligned}
y_{\lambda}=e^{\frac{\alpha_{\lambda} x^{k+1}}{k+1}+\ldots} x^{\rho_{\lambda}} & \left(C_{\lambda}+\frac{C_{\lambda 1}}{x}+\ldots+\frac{C_{\lambda n}}{x^{n}}+\frac{\gamma_{\lambda n}}{x^{n}}\right), \\
& \lim r_{\lambda n}=0 .
\end{aligned}
$$

Für eine lineare Differentialgleichung erster Ordnung

$$
\begin{gathered}
\frac{d y}{d x}+x^{k} P y=0, \\
P=a+\frac{a_{1}}{x}+\ldots+\frac{a_{n+k+1}}{x^{n+k+1}+\frac{\bar{\omega}_{n+k+1}}{x^{n+k+1}}, \quad \lim \bar{\omega}_{n+k+1}=0}
\end{gathered}
$$

ist dieser Satz gültig; denn die Integration ergibt

$$
\begin{gathered}
y=e^{-\frac{a x^{k+1}}{k+1}-\ldots} x^{a_{k+1}}\left(C+\frac{C_{1}}{x}+\ldots+\frac{C_{n}}{x^{n}}+\frac{\check{\zeta}_{n}}{x^{n}}\right), \\
\lim \gamma_{n}=0 .
\end{gathered}
$$

${ }^{1}$ Vgl. Poincare, Acta math. Bd. 8. 
Wir nehmen an, der ausgesprochene Satz sei für eine Differentialgleichung $(m-1)^{\text {ter }}$ Ordnung richtig, und zeigen, dass er dann auch für eine Differentialgleichung $m^{\text {ter }}$ Ordnung gilt.

Wir beginnen mit dem Beweis des Satzes, den wir für eine Differentialgleichung $(m-\mathrm{I})^{\text {ter }}$ Ordnung als gültig voraussetzen:

Ist in der Differentialgleichung (C)

$$
\begin{gathered}
P_{\lambda}=a_{\lambda}+\frac{a_{\lambda 1}}{x}+\ldots+\frac{a_{\lambda, n+k+1}}{x^{n+k+1}}+\frac{\bar{\omega}_{\lambda, n+k+1}}{x^{n+k+1}}, 1 \\
\lim \bar{\omega}_{\lambda, n+k+1}=0
\end{gathered}
$$

so besitzt dieselbe ein Fundamentalsystem

$$
\begin{gathered}
y_{\lambda}=e^{\frac{\alpha_{\lambda} \lambda^{k+1}}{k+1}+\ldots} x^{\rho_{\lambda}}\left(C_{\lambda}+\frac{C_{\lambda 1}}{x}+\ldots+\frac{C_{\lambda n}}{x^{n}}+\frac{\gamma_{\lambda n}}{x^{n}}\right), \\
\lim \gamma_{\lambda, n}=0 .
\end{gathered}
$$

Wir verstehen unter $y_{1}$ das in $\S 2$ nachgewiesene Integral von (C) und setzen

$$
y=y_{1} \int z d x
$$

Dann genügt $z$ der linearen Differentialgleichung $(m-\mathrm{I})^{\text {ter }}$ Ordning

$$
\frac{d^{m-1} z}{d x^{m-1}}+x^{k} Q_{1} \frac{d^{m-2} z}{d x^{m-2}}+\ldots+x^{(m-1) k} Q_{n-1} z=0 ;
$$

dabei ist, wenn man

$$
y_{1}^{(\nu)}=\frac{d^{\nu} y_{1}}{d x^{\nu}}
$$

setzt,

$$
\begin{gathered}
Q_{\mu}=(m)_{\mu} x^{-\mu k} \frac{y_{1}^{(\mu)}}{y_{1}}+(m-\mathrm{I})_{\mu-1} P_{1} x^{-(\mu-1) k} \frac{y_{1}^{(\mu-1)}}{y_{1}}+\ldots+(1)_{1} P_{\mu-1} x^{-k} \frac{y_{1}^{\prime}}{y_{1}}+P_{\mu} \\
=b_{\mu}+\frac{b_{\mu 1}}{x}+\ldots+\frac{b_{\mu, n+k+1}}{x^{n+k+1}}+\frac{\omega_{\mu, n+k+1}}{x^{n+k+1}}, \\
\lim \omega_{\mu, n+k+1}=0 .
\end{gathered}
$$

Insbesondere ist

$$
b_{\mu}=(m)_{\mu} \alpha_{1}^{\mu}+(m-1)_{\mu-1} a_{1} \alpha_{1}^{\mu-1}+\ldots+(\mathrm{I})_{1} a_{\mu-1} \alpha_{1}+a_{\mu} .
$$

${ }^{1}$ Dabei ist $n$ als feste Zahl gedacht. 
Über die asymptotische Darstellung der Integrale linearer Differentialgleichungen. 301

Setzt man

$$
\begin{aligned}
& f(\alpha)=\alpha^{m}+a_{1} \alpha^{m-1}+\ldots+a_{m}, \\
& g(\beta)=\beta^{m-1}+b_{1} \beta^{m-1}+\ldots+b_{m-1},
\end{aligned}
$$

so ist

$$
f\left(\alpha_{1}+\beta\right)=\beta g(\beta)
$$

Da die charakteristische Gleichung $f(\alpha)=$ o von (C) die Wurzeln $\alpha_{1}, \ldots, \alpha_{m}$ besitzt; so sind

$$
\beta_{2}=\alpha_{2}-\alpha_{1}, \ldots, \quad \beta_{m}=\alpha_{m}-\alpha_{1}
$$

die Wurzeln der charakteristischen Gleichung $g(\beta)=0$ von $\left(D_{1}\right)$. Es ist

$$
\circ>R\left(\beta_{2}\right)>\ldots>R\left(\beta_{m}\right) \text {. }
$$

Die Differentialgleichung $\left(\mathrm{D}_{1}\right)$ hat nach dem für die Ordnung $m$ - I vorausgesetzten Satze die $m-1$ Integrale

$$
\begin{gathered}
z_{\lambda}=e^{\frac{\beta_{\lambda} x^{k+1}}{k+1}+\frac{\beta_{\lambda \lambda^{x^{k}}}}{k}+\ldots+\beta_{\lambda k^{x}}} x^{\sigma_{k}}\left(D_{\lambda}+\frac{D_{\lambda 1}}{x}+\ldots+\frac{D_{\lambda n}}{x^{n}}+\frac{\delta_{\lambda n}}{x^{n}}\right) \\
\lim \partial_{\lambda n}=0 . \\
(\lambda=2, \ldots, m)
\end{gathered}
$$

Wir untersuchen die folgenden $m-\mathrm{I}$ Integrale von (C):

$$
y_{\lambda}=y_{1} \int_{\infty}^{x} z_{\lambda} d x
$$

Der Index $\lambda$ wird vorübergehend weggelassen.

Durch Integration der Gleichung

$$
\begin{gathered}
d\left[e^{\frac{\beta x^{k+1}}{k+1}+\frac{\beta_{1} x^{k}}{k}+\ldots+\beta_{k} x} x^{\sigma-k-\nu}\right] \\
=\left(\beta+\frac{\beta_{1}}{x}+\ldots+\frac{\beta_{k}}{x^{k}}+\frac{\sigma-k-\nu}{x^{k+1}}\right) e^{\frac{\beta x^{k+}}{k+1}+\ldots} x^{\sigma-\nu} d x
\end{gathered}
$$

zwischen den Grenzen $\infty$ und $x$ erhält man, wenn man

$$
\eta_{\nu}=\int_{\infty}^{x} e^{\frac{\beta x^{k+1}}{k+1}+\ldots} x^{\sigma-\nu} d x
$$


setzt, die Recursionsformel

$$
\beta \eta_{\nu}+\beta_{1} \eta_{\nu+1}+\ldots+\beta_{k} \eta_{\nu+k}+(\sigma-k-\nu) \eta_{\nu+k+1}=e^{\frac{\beta x^{k+1}}{k+1}+\ldots} x^{\sigma-k-\nu} .
$$

Wenn man vermittelst dieser Formel $\eta, \eta_{1}, \ldots, \eta_{n}$ durch $\eta_{n+1}, \ldots, \eta_{n+k+1}$ ausdrückt, hat man

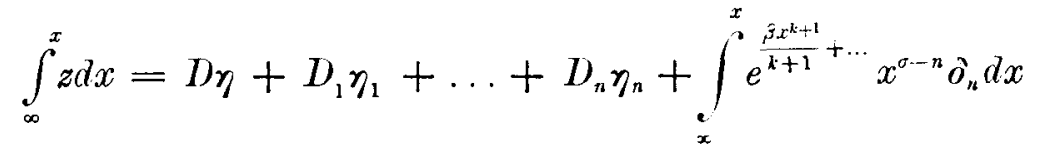

$$
\begin{aligned}
& =e^{\frac{\beta x^{k+1}}{k+1}+\ldots} x^{\sigma-k}\left(E+\frac{E_{1}}{x}+\ldots+\frac{E_{n}}{x^{n}}\right)+h_{1} \eta_{n+1}+\ldots+h_{k+1} \eta_{n+k+1} \\
& +\int_{\infty}^{x} e^{\frac{\beta x^{k+1}}{k+1}+\cdots} x^{\sigma-n} \partial_{n} d x
\end{aligned}
$$

Setzt man

$$
\tau_{n}=x \grave{o}_{n}+h_{1}+\frac{h_{2}}{x}+\ldots+\frac{h_{k+1}}{x^{k}}
$$

so ist $\lim \tau_{n}$ endlich, und die letzten Glieder des Ausdrucks für $\int_{\infty}^{x} z d x$ schreiben sich

$$
\int_{\infty}^{x} e^{\frac{\beta x^{k+1}}{k+1}+\ldots} x^{\sigma-n} \tau_{n} d x
$$

Unter Einführung der Bezeichnung

$$
\varepsilon_{n}=e^{-\frac{\hat{s} x^{k+1}}{k+1}+\ldots} x^{-\sigma+k+n} \int_{\infty}^{x} e^{\frac{\hat{\beta} v^{k+1}}{k+1}+\cdots} v^{\sigma-n-1} \tau_{n}(v) d x
$$

hat man

$$
\int_{\infty}^{x} z d z=e^{\frac{\beta x^{k+1}}{k+1}+\ldots} x^{\sigma-k}\left(E+\frac{E_{1}}{x}+\ldots+\frac{E_{n}}{x^{n}}+\frac{\varepsilon_{n}}{x^{n}}\right) .
$$

Um zu beweisen, dass

$$
\lim \varepsilon_{n}=0
$$

ist, setzt man

$$
v^{k+1}=x^{k+1}+w
$$


Über die asymptotische Darstellung der Integrale linearer Differentialgleichungen. 303 oder

$$
v=x\left(1+\frac{w}{x^{k+1}}\right)^{\frac{1}{k+1}}
$$

so dass $\varepsilon_{n}$ übergeht in

$$
\begin{aligned}
\varepsilon_{n}=- & \frac{\mathrm{I}}{(k+\mathrm{I}) x} \int_{0}^{\infty} e^{\frac{\beta w}{k+1}+\sum_{\nu=1}^{k} \frac{\beta_{t}+1+\nu}{\nu}\left[\left(1+\frac{w}{x^{k+1}}\right)^{\frac{\nu}{k+1}}-1\right]} \\
& \times\left(\mathrm{I}+\frac{w}{x^{\bar{k}+l}}\right)^{\frac{\sigma-n-k-1}{k+1}} \tau_{n}(v) d w
\end{aligned}
$$

Für $t \geq 0$ ist

$$
\underbrace{\left|\beta_{k+1-\nu}\right|}_{\nu} \frac{\left(\mathrm{I}+t^{\frac{k+1}{\nu}}\right)^{\frac{\nu}{k+1}}-1}{t}
$$$$
(y=1, \ldots, k)
$$

positiv und kleiner als $M_{\nu}$; setzt man

$$
t=\frac{w^{\frac{\nu}{k+1}}}{x^{\nu}}
$$

so hat man für $w \geqq 0, x>0$

$$
\frac{\left|\beta_{k+1-\nu}\right|}{\nu} x^{\nu}\left[\left(1+\frac{w}{x^{k+1}}\right)^{\frac{\nu}{k+1}}-\mathrm{I}\right]<M_{\nu} w^{\frac{\nu}{k+1}}
$$

Ferner ist für $w \geq 0, x>$ I

$$
\frac{|\sigma-n-k-\mathrm{I}|}{k+\mathrm{I}} \log \left(\mathrm{I}+\frac{w}{x^{k+1}}\right)<M \log (\mathrm{I}+w),
$$

wo $M$ eine positive Grösse ist. Wenn man $x$ so gross nimmt, dass $\left|\tau_{n}(x)\right|$ und demnach auch $\left|\tau_{n}(v)\right|$ unterhalb der endlichen Grösse $g$ liegt, so ist

$$
\left|s_{n}\right|<\frac{g}{(k+\mathrm{I}) x} \int_{0}^{\infty} e^{\frac{R(\beta) w}{x^{k+1}}+\sum_{k}^{\nu=1} M_{v} w^{\frac{\nu}{k+1}}+M \operatorname{lng}(1+w)} d w .
$$

Das letzte Integral ist endlich und daher

$$
\lim \varepsilon_{n}=\text { o. }
$$


Wenn man den Index $\lambda$ wieder einführt, hat man

$$
\begin{gathered}
\int_{\infty}^{x} z_{\lambda} d x=e^{\frac{\beta_{\lambda} x^{k+1}}{k+1}+\ldots} x^{\sigma_{\lambda}-k}\left(E_{\lambda}+\frac{E_{\lambda 1}}{x}+\ldots+\frac{E_{\lambda n}}{x^{n}}+\frac{\varepsilon_{\lambda n}}{x^{n}}\right), \\
\lim \varepsilon_{\lambda n}=0
\end{gathered}
$$

also

$$
\begin{array}{r}
y_{\lambda}=e^{\frac{a_{1} x^{k+1}}{k+1}+\cdots} x^{\rho_{1}}\left(C_{1}+\frac{C_{11}}{x}+\ldots+\frac{C_{1 n}}{x^{n}}+\frac{\gamma_{1 n}}{x^{n}}\right) \\
\times e^{\frac{\beta_{\lambda x^{k+1}}^{k+1}}{k+\ldots} x^{\sigma_{\lambda}}}\left(E_{\lambda}+\frac{E_{\lambda 1}}{x}+\ldots+\frac{E_{\lambda n}}{x^{n}}+\frac{\varepsilon_{\lambda n}}{x^{n}}\right)
\end{array}
$$

oder für $\lambda=2, \ldots, m$

$$
\begin{gathered}
y_{\lambda}=e^{\frac{\alpha_{\lambda} x^{k+1}}{k+1}+\frac{a_{\lambda 1} x^{k}}{k}+\ldots+a_{\lambda, k} x} x^{\rho_{\lambda}}\left(C_{\lambda}+\frac{C_{\lambda 1}}{x}+\ldots+\frac{C_{\lambda n}}{x^{n}}+\frac{\gamma_{\lambda n}}{x^{n}}\right), \\
\lim r_{\lambda n}=0 .
\end{gathered}
$$

Ferner hat man

$$
x^{-k} \frac{d \log y_{\lambda}}{d x}=x^{-k} \frac{d \log y_{1}}{d x}+\frac{z_{\lambda}}{x^{k} \int_{\infty}^{x} z_{\lambda} d x},
$$

woraus unter Einsetzung der bekannten Entwicklungen für die Grössen auf der rechten Seite hervorgeht:

$$
\begin{gathered}
x^{-k} \frac{d \log y_{\lambda}}{d x}=\alpha_{\lambda}+\frac{\alpha_{\lambda 1}}{x}+\ldots+\frac{\alpha_{\lambda, n+k}}{x^{n+k}}+\frac{\eta_{\lambda n}}{x^{n+k}}, \\
\lim \eta_{\lambda n}=0 .
\end{gathered}
$$

$\S 4$.

Wir haben gesehen, dass die Differentialgleichung (C) $m$ Integrale

mit der Eigenschaft

$$
y_{1}, \ldots, y_{m}
$$

$$
\lim x^{-k} \frac{d \log y_{\grave{\lambda}}}{d x}=\alpha_{\lambda}
$$


Über die asymptotische Darstellung der Integrale linearer Differentialgleichungen. 305

oder

$$
y_{\lambda}=e^{\left(a_{\lambda}+\delta_{\lambda}\right) \frac{x^{k+1}}{k+1}}, \quad \lim \delta_{\lambda}=0 \quad(\lambda=1, \ldots, m)
$$

besitzt. Aus dieser Form geht hervor, dass eine Relation

$$
\sum_{\lambda} c_{\lambda} y_{\lambda}=0
$$

mit constanten Coefficienten nicht bestehen kann, dass also $y_{1}, \ldots, y_{m}$ ein Fundamentalsystem bilden.

Ist

$$
\bar{y}_{m}=c_{1} y_{1}+\ldots+c_{m} y_{m}
$$

irgend ein Integral mit der Eigenschaft

$$
\lim x^{-k} \frac{d \log \bar{y}_{m}}{d x}=\alpha_{m},
$$

so muss $c_{1}=0, \ldots, c_{m-1}=0$ sein; es gibt also nur ein einziges Integral $y_{m}$, für welches

$$
\lim x^{-k} \frac{d \log y_{m}}{d x}=a_{m}
$$

ist, wenn Integrale, welche sich bloss um einen constanten Factor unterscheiden, als identisch angesehen werden.

Das Integral $y_{m}$ von (C) ist folgendermassen entstanden. Nach Festlegung der ganzen positiven Zahl $n$ ist $\zeta_{\lambda}=\zeta_{\lambda, n+k+1}(\lambda=2, \ldots, m)$ irgend ein Integralsystem des in $\S 1$ aufgestellten, aus (C) auf die in $\S 2$ angegebene Weise hergeleiteten Differentialgleichungssystems mit der Eigenschaft

$$
\lim \zeta_{\lambda}=0
$$$$
(\lambda=2, \ldots, m)
$$

Die Gleichung

$$
y_{1}=e^{\int \frac{a_{1}+a_{2} z_{3}+\ldots+a_{m} z_{m}}{1+z_{2}+\ldots+z_{m}} d x}
$$

wo

$$
z_{\lambda}=\frac{c_{\lambda 1}}{x}+\ldots+\frac{c_{\lambda, n+k+1}}{x^{n+k+1}}+\frac{\zeta_{\lambda, n+k+1}}{x^{n+k+1}}
$$


ist, stellt ein Integral von (C) dar. Wenn unter $z_{m}$ das einzige Integral von $\left(\mathrm{D}_{1}\right)$ verstanden wird, für welches

$$
\lim x^{-k} \frac{d \log z_{m}}{d x}=\beta_{m}=\alpha_{m}-\alpha_{1}
$$

ist, so ist

$$
y_{m}=y_{1} \int_{\infty}^{x} z_{m} d x
$$

Setzt man an Stelle der ursprünglich angenommenen Zahl $n$ irgend eine ganze positive Zahl $n^{\prime}>n$, während im übrigen das bei der Bildung von $y_{m}$ benutzte Verfahren beibehalten wird, so stösst man auf die Function

welche, weil

$$
y_{m}^{\prime}=e^{\frac{\alpha_{m} x^{k+1}}{b+1}+\ldots} x^{o_{m}}\left(C_{m}+\frac{C_{m 1}}{x}+\ldots+\frac{C_{m n^{\prime}}}{x^{n^{\prime}}}+\frac{\gamma_{m n^{\prime}}}{x^{n^{\prime}}}\right)
$$

$$
\lim \gamma_{m n^{\prime}}=\mathrm{O}
$$

$$
\lim x^{-k} \frac{d \log y_{m}^{\prime}}{d x}=\alpha_{m}
$$

ist, mit $y_{m}$ identisch sein muss.

Es ist als für jeden Werth ron $n$

$$
\begin{gathered}
y_{n}=e^{\frac{a_{m} x^{k+1}}{k+1}+\ldots} x^{\rho_{m}}\left(C_{m}+\frac{C_{m_{1}}}{x}+\ldots+\frac{C_{m n}}{x^{n}}+\frac{\gamma_{m n}}{x^{n}}\right) \\
\lim \gamma_{m n}=0 .
\end{gathered}
$$

$\S 5$.

Wir wissen jetzt, dass das Integral $y_{m}$ durch die Normalreihe

$$
S_{m}=e^{\frac{a_{m} x^{2}+1}{k+1}+\ldots} x^{\rho_{m}}\left(C_{m}+\frac{C_{m 1}}{x}+\frac{C_{m 2}}{x^{2}}+\ldots\right)
$$

asymptotisch dargestellt wird.

Es ist noch zu zeigen, dass für jeden Werth $\lambda=1, \ldots, m$ das Integral $y_{\lambda}$ durch die Normalreihe $S_{\lambda}$ asymptotisch dargestellt wird, d. h. dass für jeden Werth von $n$

$$
y_{\lambda}=e^{\frac{\alpha_{\lambda} x^{k+1}}{k+1}+\cdots} x_{\lambda}^{\rho_{\lambda}}\left(C_{\lambda}+\frac{C_{\lambda 1}}{x}+\ldots+\frac{C_{\lambda, n}}{x^{n}}+\frac{\gamma_{\lambda n}}{x^{n}}\right), \quad \lim \gamma_{\lambda n}=0
$$


Über die asymptotische Darstellung der Integrale linearer Differentialgleichungen. 307 ist, nicht nur für die in $\S 2$ eingeführte feste /ahl $n$. Wir verstehen unter $n^{\prime}$ eine beliebige ganze positive Zahl, welche die ursprünglich eingeführte Zahl $n$ übertrifft. Wir bilden, von dieser Zahl $n^{\prime}$ ausgehend, ein Integral $y_{\lambda}^{\prime}$ ebenso, wie $y_{\lambda}$ mit der Zahl $n$ gebildet wurde. Dann ist

$$
\begin{gathered}
y_{\lambda}^{\prime}=e^{\frac{\alpha_{\lambda} x^{k+1}}{k+1}+\ldots} x^{\rho_{\lambda}}\left(C_{\lambda}+\frac{C_{\lambda 1}}{x}+\ldots+\frac{C_{\lambda n^{\prime}}}{x^{n^{\prime}}}+\frac{\gamma_{\lambda n^{\prime}}}{x^{n^{\prime}}}\right), \\
\\
\lim \gamma_{\lambda n^{\prime}}=0 .
\end{gathered}
$$

Die Integrale $y_{1}^{\prime}, \ldots, y_{m}^{\prime}$ bilden ein Fundamentalsystem; es ist

$$
y_{\lambda}=y_{\lambda}^{\prime}+c_{\lambda+1} y_{\lambda+1}^{\prime}+\ldots+c_{m} y_{m}^{\prime} \text {. }
$$

Wenn man $y_{\lambda}^{\prime}$ durch den obigen Ausdruck ersetzt und

$$
y_{\mu}^{\prime}=e^{\frac{\alpha_{\mu} x^{k+1}}{k+1}+\ldots} x^{\rho_{\mu}}\left(C_{\mu}+\gamma_{\mu}^{\prime}\right), \quad \lim \gamma_{;}^{\prime}=0 \quad(\mu=\lambda+1, \ldots, m
$$

setzt, so hat man

$$
\begin{aligned}
y_{\lambda}= & e^{\frac{\alpha_{\lambda} x^{k+1}}{k+1}+\ldots} x^{\rho_{\lambda}}\left(C_{\lambda}+\frac{C_{\lambda 1}}{x}+\ldots+\frac{C_{\lambda n^{\prime}}}{x^{n^{\prime}}}+\frac{\gamma_{\lambda n^{\prime}}^{\prime}}{x^{n^{n}}}\right) \\
& +\sum_{\mu=\lambda+1}^{m} e^{\frac{\left(\alpha_{\mu}-\alpha_{\lambda}\right) x^{k+1}}{k+1}+\ldots} x^{\rho^{\rho^{2}-\rho_{\lambda}}} c_{\mu}\left(C_{\mu}+\gamma_{\mu}^{\prime}\right) .
\end{aligned}
$$

Setzt man

$$
\gamma_{\lambda n^{\prime}}=\gamma_{\lambda n^{\prime}}^{\prime}+\sum_{\mu=i+1}^{m} e^{\frac{\left({ }^{\alpha}-a_{\lambda}\right) x^{k+1}}{r+1}+\ldots} x^{\rho^{\rho^{\prime}-\rho_{\lambda}+n}} c_{\mu}\left(C_{\mu}+\gamma_{\mu}^{\prime}\right)
$$

so ist, da der reelle Theil von $\alpha_{\mu}-\alpha_{\lambda}$ negativ ist,

und man hat

$$
\lim \gamma_{\lambda n^{\prime}}=0
$$

$$
y_{\lambda}=e^{\frac{a_{\lambda} x^{k+1}}{k+1}+\ldots} x^{o_{\lambda}}\left(C_{\lambda}+\frac{C_{\lambda 1}}{x}+\ldots+\frac{C_{\lambda n^{\prime}}}{x^{n^{\prime}}}+\frac{\gamma_{\lambda n^{\prime}}}{x^{n^{\prime}}}\right)
$$

für jede Zahl $n^{\prime}$, w. z. b. w.

Ist

$$
y=c_{1} y_{1}+\ldots+c_{m} y_{m}
$$

irgend ein Integral von (C) und

$$
c_{1}=\ldots=c_{\lambda-1}=0, \quad c_{\lambda} \neq 0
$$


so hat man für einen beliebigen Werth von $n$

$$
y=c_{\lambda} e^{\frac{a_{\lambda} x^{k+1}}{k+1}+\ldots} x^{\rho_{\lambda}}\left(C_{\lambda}+\frac{C_{\lambda_{1}}}{x}+\ldots+\frac{C_{\lambda n}}{x^{n}}+\frac{\gamma_{\lambda n}}{x^{n}}\right)+\sum_{\mu=\lambda+1}^{m} c_{\mu} e^{\frac{\left(a_{\mu}+\delta_{\mu}\right) x^{k+1}}{k+1}}
$$

wo

$$
\lim r_{\lambda n}=0, \quad \lim \delta_{\mu}=0
$$

ist. Setzt man

$$
\bar{r}_{\lambda n}=\gamma_{\lambda n}+\sum_{\mu=\lambda+1}^{m} \frac{c_{\mu}}{c_{\lambda}} e^{\left(a_{\mu}-\alpha_{\lambda}+\delta_{\mu}\right) \frac{x^{k}+1}{k+1}-\frac{a_{\lambda_{1}} z^{k}}{k}-\cdots} x^{-\rho_{\lambda}+n}
$$

so ist

$$
y=c_{\lambda} e^{\frac{a_{\lambda} x^{k+1}}{k+1}+\ldots} x^{\rho \lambda}\left(C_{\lambda}+\frac{C_{\lambda 1}}{x}+\ldots+\frac{C_{\lambda n}}{x^{n}}+\frac{\bar{\gamma}_{\lambda n}}{x^{n}}\right)
$$

d. h. das Integral

$$
\lim \vec{\gamma}_{\lambda n}=0 \text {; }
$$

$$
y=c_{\lambda} y_{\lambda}+\ldots+c_{m} y_{m}
$$

wird, wenn $c_{\lambda}$ von Null verschieden ist, durch die Reihe $c_{\lambda} S_{\lambda}$ asymptotisch dargestellt.

Wir haben uns bisher auf die Betrachtung reeller positiver Werthe von $x$ beschränkt. Geht $x$ mit dem Argument $\omega$ ins Unendliche, so wird die Differentialgleichung (C) durch die Substitution $x=\rho e^{i \omega}$ in eine Differentialgleichung mit der unabhängigen Veränderlichen $\rho$ übergeführt, deren charakteristische Gleichung die Wurzeln

$$
e^{i(\boldsymbol{k}+1) \omega} \alpha_{1}, \ldots, e^{i(\boldsymbol{k}+1) \omega} \alpha_{m}
$$

besitzt. Durch die bisherige Voraussetzung, dass die reellen Theile der Wurzeln der charakteristischen Gleichung verschieden sind, sind diejenigen Argumente vorläufig ausgeschlossen, für welche zwei der Grössen $e^{i(k+1)} \alpha_{\lambda}$ gleiche reelle Theile erhalten. 